\title{
Gradenigo's Syndrome a Rare Complication of Acute Otitis Media: Case Report and Literature Revue
}

\author{
Case Monia Ghammam, Amel Gdissa, Mouna Bellakhdher, Jihene Houas and \\ Report \\ Mohamed Abdelkefi \\ Department of ENT and Cervical Surgery Farhat Hached Hospital, Medicine University \\ Sousse, Tunisia
}

\begin{abstract}
Introduction: Gradenigo's syndrome is a rare complication of otitis media and mastoiditis involving the apex of the petrous temporal bone. This syndrome is characterized by a clinical triad of deep facial pain, sixth cranial nerve palsy, and acute otitis media (AOM).

Case report: We report a case of a six-year-old girl, with no past medical history, who presented with a sudden diplopia following six days of headache and otorrhea. The diagnosis of gradenigo's syndrome was confirmed by clinical examination and imaging. She made a full recovery after 9 weeks of antibiotics.

Discussion: Gradenigo's syndrome is a rare but life threatening complication of AOM.

The inflammatory process starts in the middle ear, and spreads from the base to the top of the petrous portion of the temporal bone, producing several diagnostic symptoms.

Careful clinical history and physical examination, including imaging, are necessary to make the diagnosis.

Appropriate management requires antibiotic treatment and possible surgical intervention.
\end{abstract}

Key Words: Abducens nerve palsy, antibiotics, facial pain, Gradenigo's syndrome, otitis media.

Received: $23^{\text {rd }}$ January 2019, Accepted: $13^{\text {th }}$ October 2019

Corresponding Author: Monia Ghammam, Department of ENT, Surgery Farhat Hached Hospital, Medicine University Sousse, Tunisia, Tel.: +216 52308755, E-mail: ghammamonia@gmail.com.

ISSN: 2090-0740, January 2020 Vol. 21, No. 1.

\section{INTRODUCTION}

Prior to the introduction of widespread antibiotics, apical petrositis was a fatal complication of otitis media.

In 1904, Gradenigo described a triad of symptoms related to petrous apicitis, including abducens nerve palsy, pain in the distribution of the fifth cranial nerve and suppurative acute otitis media ${ }^{[1]}$. Only 42 per cent of his patients presented the full symptoms. The signs and symptoms of the disease process are often indistinct, and presentations vary enormously.

These cranial nerve dysfunctions are caused by osteitis of the petrous apex (petrous apicitis) and are very rare complications of otitis media, especially since the widespread use of antibiotics. However, in recent years, rises in the incidence of intratemporal and intracranial complications of purulent middle ear infections have been mentioned in the literature, mainly in younger children.

The trigeminal nerve ganglion and the abducens nerve are separated from the petrous apex only by dura mater and are therefore vulnerable to any inflammatory process occurring in this region.

The inflammatory process starts in the middle ear, and spreads from the base to the top of the petrous portion of the temporal bone, producing several diagnostic symptoms.

\section{CASE REPORT}

A 6-year-old girl, fully vaccinated, who was in a good health with no past medical history of recurrent AOM, was admitted to our hospital for sudden onset of diplopia following 6 days of headaches, right otalgia and otorrhea. She had been treated with orally administered antibiotics for otitis media 3 days before the onset of these symptoms.

At admission she had right otalgia and otorrhea, with bulging perforated tympanic membrane. The left tympanic membrane was normal. There was no mastoid tenderness. Her temperature was 37.7. She had no meningeal signs. Examination of eyeball movement revealed right abductor nerve palsy (Figure 1). 

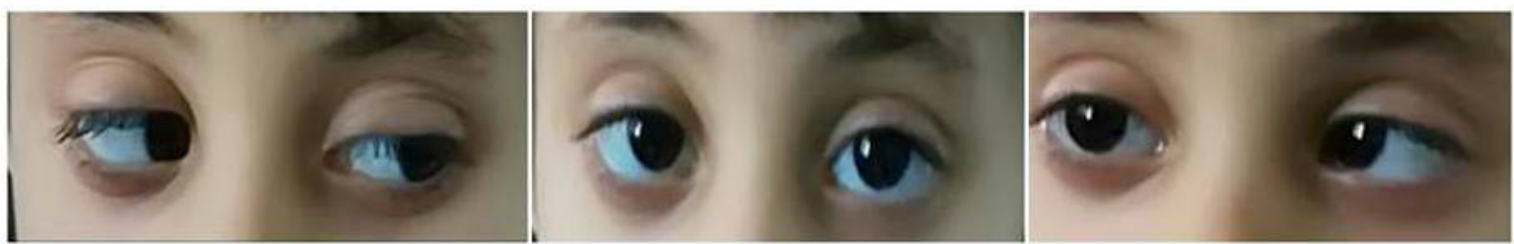

Fig. 1: Right abducens nerve palsy.

There was a normal facial motility. Facial pain was localized in the first and second branches of the right trigeminal nerve. Ophtalmoscopy and biomicroscopy were normal. The diagnosis of petrous apicitis was suspected.

Audiometry revealed a $30 \mathrm{~dB}$ right side conductive hearing loss.
Laboratory tests showed a white blood cell count of $4800 / \mathrm{UL}$, a platelet count of $364000 / \mathrm{uL}$, a sedimentation rate of 40 and a C-reactive protein of $8 \mathrm{mg} / \mathrm{L}$.

A CT scan of the brain and the petrous temporal bone showed a subtotal filling of the right middle ear, and the cavernous sinus was clear (Figure 2).

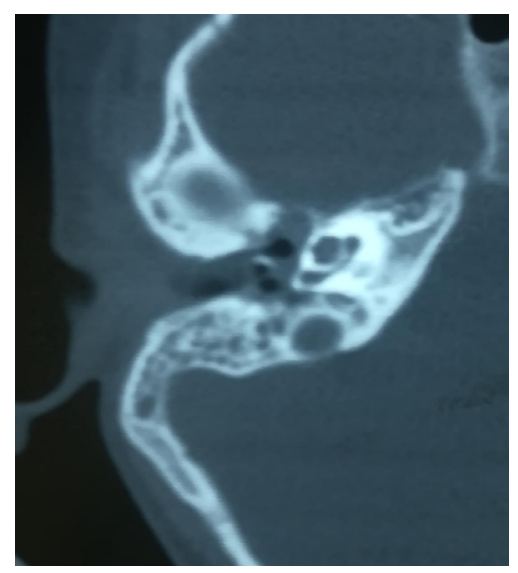

Fig. 2: CT scan of the brain and the petrous temporal bone showed a subtotal filling of the right middle ear.

MRI of her head showed a mass lesion of the right petrous apex and evidence of inflammatory disease of middle ear cavity (Figure 3).

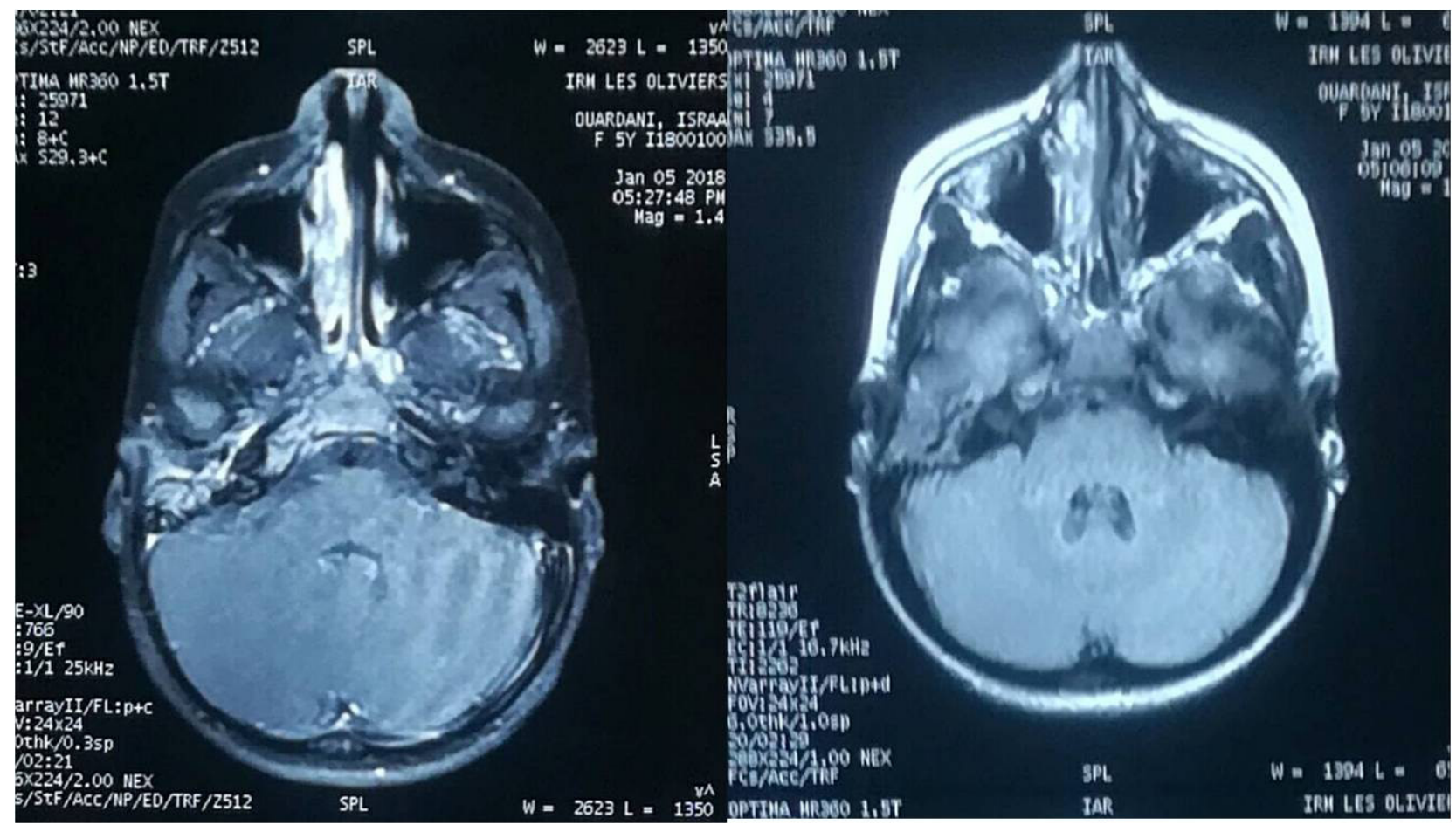

Fig. 3: Brain Magnetic Resonance Imaging (MRI) findings at admission: T2 weighted MRI finding (A) and T1 weighted enhanced MRI finding (B). These showed high signal intensities involving right mastoid and petrous apex. 
She was commenced on $200 \mathrm{mg} / \mathrm{kg} / 24 \mathrm{~h} \mathrm{IV} \mathrm{co-amoxiclav} \mathrm{and} 2 \mathrm{mg} / 24 \mathrm{~h}$ dexamethason. The patient completed 6 weeks of intravenous antibiotics and 3 weeks of oral antibiotics, with favorable outcome. She made complete recovery after 9 weeks (Figure 4).

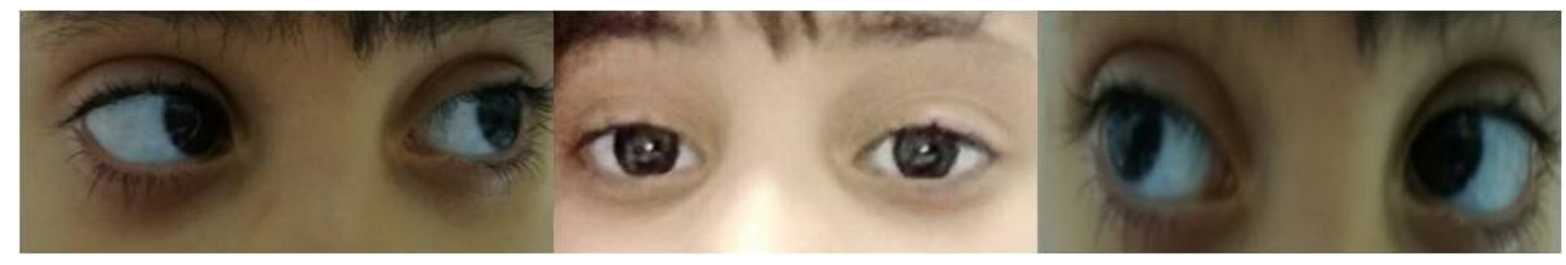

Fig. 4: A normal ocular motility

\section{DISCUSSION AND REVIEW OF LITERATURE}

Gradenigo's syndrome is a rare but life threatening complication of $\mathrm{AOM}^{[2,3]}$

Clinically, it is characterized by the triad of suppurative otitis media, deep facial pain, and abducens palsy. Most of the Gradenigo syndrome cases that have been reported in the literature were caused by pyogenic bacteria ${ }^{[4]}$.

It is commonly caused by aerobic bacteria. The most frequently isolated organisms are Streptococcus pneumonia, beta hemolytic streptococci, staphylococcus species, hemophylus inflenzae, Pseudomonas species,Moraxalla catarhalis $^{[2,5]}$ and klebsielle pneumonia ${ }^{[6]}$. But anaerobic bacteria such as F.necrophorum can be causative pathogen as well[ ${ }^{[7]}$.

Among the eight patients with Gradenigo's syndrome studied by Chole and Donald in 1983, they isolated a causative agent in six: Five Psudomonas species, and one streptococcus pneumonia ${ }^{[8]}$

The diagnosis of petrous apicitis has become easier with the revolutions of imaging technology.

CT scanning remains the first line investigation for possible lesions of the petrous apex because it is more available than MRI. It is also sensitive enough in detecting bone erosion and demonstrating the extent of temporal bone pneumatization. Besides, it is able to detect intracranial abcess formation. But it is less sensitive than MRI in detecting early intracranial complications ${ }^{[8]}$.

MRI is used to detect the extent of meningeal and cerebral involvement. It is utilized also to discern between the differing lesions featuring in the petrous apex, other than petrous apicitis, such as osteomyelitis, cholesteatoma, and neoplasic lesions ${ }^{[8,9]}$.

The advent of the antibiotic era has not only made the incidence of such fatal AOM'S complication become rare, but has also facilitated the conservative management of a select cohort of patients with petrous apicitis, a disease that has been historically managed with surgical intervention. Surgical debridement is only required in a few and severe complicated cases with abscesses or osteomyelitis ${ }^{[10]}$.

Our patient with the Gradenigo's syndrome triad was treated without the recourse to surgical management ${ }^{[8]}$.
In the light of the inconstant infectious pattern of the organism and its antibiotic resistance profile that is continually being modified, it remains impossible to recommend an all encompassing strategy for antibiotic usage in the treatment of petrous apicitis.

Two associations were indicated in the literature: Ceftriaxone $50 \quad \mathrm{mg} / \mathrm{kg} / 24 \mathrm{~h}$ and metronidazole $40 \mathrm{mg} / \mathrm{kg} / 24 \mathrm{~h}$ in association with vancomycin for an average of 30 days, or with amikacin $3 \mathrm{mg} / \mathrm{kg} / 24 \mathrm{~h}$ for 3 days.

The antibiotheray was relayed with oral amoxi clav for 3 to 4 weeks.

There is no information about the delay of the resolution of abducens nerve palsy. Published case reports of apical petrositis associated with sixth nerve palsy remain very few. Authors have reported variable times for resolution of the lateral rectus paralysis from 10 to 64 days $^{[10]}$.

Gillanders described the resolution of lateral rectus palsy 6 weeks after posterior petrosectomy and high-dose antibiotics of a 40 year old patient with petrous apicitis ${ }^{[11]}$.

Price and Fayed detected that the sixth nerve palsy subsides after only 9 days after attici-antrectomy and antibiotherapy of a 7 year old patient who was noted to have Gradenigo's syndrome ${ }^{[9]}$.

Hilding and Price reported improvement in the sixth nerve palsy of a 9 month old patient with mastoiditis in 11 days after mastoidectomy with intra venous antibiotics ${ }^{[12]}$.

Minotti and Kauntaki studied the outcomes of two patients presenting with petrositis and abducens palsy: one after an AOM, the other developing it bilaterally after a chronic bilateral otitis media ${ }^{[5,13]}$.

They noticed that the acute form responded well to conservative management with antibiotics associated to myringotomy or tube tympanotomy. A complete resolution was detected within 4 days of antibiotherapy. The chronic condition failed to respond to conservative management, and proceeded to surgical decompression of petrous apex, with resolution of the sixth nerve within 5 days.

\section{CONCLUSION}

Although it is a rare condition, Gradenigo's syndrome 
remains a fatal complication of otitis media that should be looked for and treated in order to avoid intracranial complications such as brain abscess.

CT scan and MRI represent the top diagnosis arsenal.

Conservative management is possible with the evolution of antibiotics, and early diagnosis.

\section{CONFLICT OF INTEREST}

There are no conflicts in interest.

\section{REFERENCES}

1. Taklalsingh N, Falcone F, Velayudhan V. Gradenigo's syndrome in a patient with chronic suppurative otitis media, petrous apicitis, and meningitis. Am J Case Rep. 2017;18:1039-43.

2. Heshin-Bekenstein $\mathrm{M}$, Megged $\mathrm{O}$, Peleg $\mathrm{U}$, Shahroor-Karni S, Bass R, Benifla $\mathrm{M}$, et al. Gradenigo's syndrome: Is fusobacterium different? Two cases and review of the literature. Int J Pediatr Otorhinolaryngol. 2014;78(1):166-9.

3. Motamed M. Kalan A. Gradenigo's syndrome. Postgrad Med J. 2000;76(886):559-60.

4. Chen PY, Wu CC, Yang TL, Hsu CJ, Lin YT, Lin KN Gradenigo syndrome caused by nontuberculous mycobacterian. Audiol Neurootol. 2014;19(4):275-82.

5. Lutter SA, Kerschner JE CM. Gradenigo syndrome: A rare but serious complication of otitis media. Pediatr Emerg Care. 2005;21:384-6.

6. Mittal R, Lisi CV GR et al. Current concepts in the pathogenesis and treatment of chronic suppurative otitis media. J Med Microbiol. 2015;64:1103-16.

7. Jacobsen C Bruhn M Yavarian Y Gaihede $M$. Mastoiditis and Gradenigo's Syndrome with anaerobic bacteria. BMC Ear Nose Throat Disord. 2012;12:10.

8. Burston BJ, Pretorius PM, Ramsden JD. Gradenigo's syndrome: successful conservative treatment in adult and paediatric patients. $\mathrm{J}$ Laryngol Otol. 2005;119 (April):325-9.

9. Price T, Fayad G. Abducens nerve palsy as the sole presenting symptom of petrous apicitis. J Laryngol Otol. 2002;116(9):726-9.

10. Jensen PV, Hansen MS, Møller MN SJ. The forgotten syndrome? Four cases of Gradenigo's syndrome and a review of the literature. Strabismus. 2016;24:21-7.

11. Gillanders DA. Gradenigo's syndrome revisited. J Otolaryngol. 1983;12:169-74.

12. Hilding DA. Petrous apex and subarcuate fossa maturation. Laryngoscope. 1987;97:1129-35.

13. Minotti AM KS. Management of abducens palsy in patients with petrositi. Ann Otol Rhinol Laryngol. 1999;108:897-902. 